\title{
Response to letter to the editor
}

\author{
Shogo Tsujikawa ${ }^{1} \cdot K_{\text {Kazutoshi Ikeshita }}{ }^{1}$
}

Received: 12 March 2019 / Accepted: 16 March 2019 / Published online: 5 April 2019

(c) Japanese Society of Anesthesiologists 2019

Keywords · Dexmedetomidine · Carotid endarterectomy $\cdot$ Hemodynamics

To the Editor:

We are grateful to the readers for their interest in our article [1] and for providing insightful comments.

We agree with the important points raised by them. It is true that the dose of nicardipine and the plasma norepinephrine level are only surrogate markers of hemodynamic stability. However, we revealed that dexmedetomidine, which was infused intraoperatively during carotid endarterectomy, one of the most high-risk surgeries, stabilized the hemodynamics because of effective analgesia and appropriate sedation levels.

High-risk patients undergoing vascular surgeries including carotid endarterectomy are associated with a high incidence of hemodynamic instability, and strict control of arterial blood pressure improves the outcome by reducing perioperative complications. Jin et al. reported that dexmedetomidine might not reduce the risk of perioperative cardiac complications in patients who underwent non-cardiac surgeries [2]. However, in this meta-analysis, they analyzed all patients undergoing low-risk as well as high-risk surgeries. The degree of surgical procedure risk including frailty scale was not estimated accurately in many studies. The effects of dexmedetomidine may be especially beneficial for high-risk patients undergoing carotid endarterectomy. We hope that our study will help further understanding of the beneficial effects of dexmedetomidine in high-risk patients.

Acknowledgements No funding was received.

\section{Compliance with ethical standards}

Conflict of interest No conflicts of interest.

\section{References}

1. Tsujikawa S, Ikeshita K. Low-dose dexmedetomidine provides hemodynamics stabilization during emergence and recovery from general anesthesia in patients undergoing carotid endarterectomy: a randomized double-blind, placebo-controlled trial. J Anesth. 2019;33:266-72.

2. Jin S, Zhou X. Influence of dexmedetomidine on cardiac complications in non-cardiac surgery: meta-analysis of randomized trials. Int J Clin Pharm. 2017;39:629-40.

Publisher's Note Springer Nature remains neutral with regard to jurisdictional claims in published maps and institutional affiliations.

This reply refers to the comment available online at https://doi. org/10.1007/s00540-019-02633-5.

Shogo Tsujikawa

seashogo@shirt.ocn.ne.jp

1 Department of Anesthesiology, Yao Tokushukai General Hospital, 1-17, Wakakusamachi, Yao, Osaka 581-0011, Japan 